\title{
Unsteady transition from a Mach to a regular shockwave intersection
}

\author{
N.C. MARKATOS, S. J. KARABELAS \\ Department of Chemical Engineering, Computational Fluid Dynamics Unit \\ National Technical University of Athens (N.T.U.A.) \\ 9, Heroon Polytechniou str., Zografou, GR-15780 \\ GREECE
}

\begin{abstract}
The purpose of this research work is to perform accurate numerical computations of supersonic flow with second order discretization of governing terms in a converging nozzle and specifically to study Mach disks. The latter process has been widely studied over the last years. In the present study numerical simulations are performed for transient supersonic flow, tracing the transition from a Mach reflection to a regular one. This has been done by enforcing the walls of a converging nozzle to come closer together, changing the deflection angle with time. Viscosity was taken into account and the full Navier-Stokes have been solved. The results obtained clearly show the gradual extinction of the Mach disk and the eventual wave intersection to a single point.
\end{abstract}

Key-Words: supersonic flow, Mach disk, shock waves, Mach intersection

Received: May 13, 2021. Revised: August 24, 2021. Accepted: September 7, 2021. Published: September 21, 2021.

\section{Introduction}

The properties that distinguish a shock wave from other waves are that its thickness is negligible compared with other characteristic lengths, and that the state of the medium is changed irreversibly by the passage of the wave. Shocks are therefore a highly nonlinear phenomenon, and parameter changes may be expected to lead to numerous bifurcations. The multitude ofpossibilities is compounded when more than one shock occurs, such as in the interaction of a shock with a solid surface or symmetry plane (i.e., in shock reflection). Interesting considerations on this topic may be found in recent references [1-3]. The subject of shock reflection is complicated and it is necessary to solve the full viscous Navier-Stokes equations to get insight to the process.

A symmetrical parabolic converging nozzle of inlet diameter $4 \mathrm{~m}$ and outlet $3 \mathrm{~m}$ was used to generate shock waves (Fig.1). According to shock reflection theory, when the shock angle $\varphi$ is smaller than the Von-Neumman critical angle $\varphi_{v}$ the shock wave reflects into a regular reflection. In our case both shock waves induced from the top and bottom walls intersect to a regular intersection (single point) [4-7]. For shock angles greater than the detachment angle $\varphi_{d}$ a Mach intersection will be induced, which means that the intersecting waves form a Mach disk $[8,9]$. In the range between $\varphi_{v}$ and $\varphi_{d}$ both types of reflection could be induced.

Despite the existence of those critical angles, the transition conditions are not very clear. There are differences between experimental and numerical results, especially in the Mach disk region and considerable disagreement between numerical and theoretical values concerning the above mentioned critical angles.

The waves' intersection closely to an axis of symmetry is examined here. At the inlet region, due to the deflection of walls, oblique shock waves are formed.
These waves intersect at two points, known as Mach or triple points, leading to a normal shock wave, the Mach disk. Afterwards these waves reflect on the walls downstream. According to theory, depending on flow conditions, there form two slip lines or discontinuity lines and a vortex sheet.

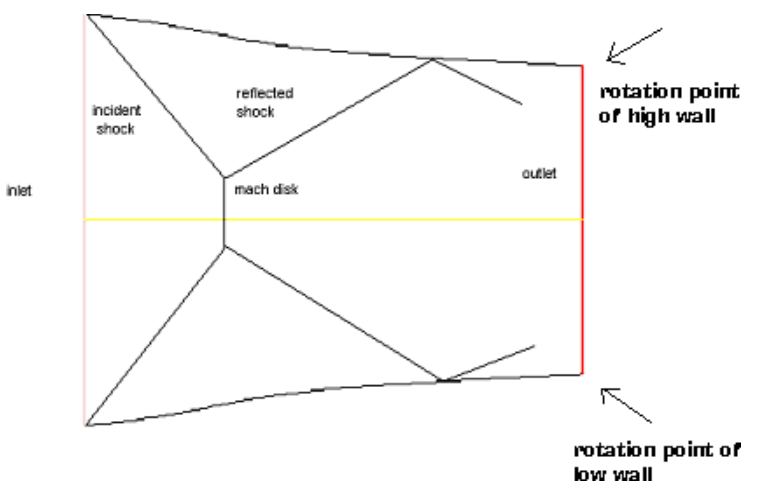

Fig. 1. Shock wave intersections, causing mach disk.

In transient problems hysteresis is observed, due to the continuous changes of the deflection angle $[10,11]$. The result is transitions from Mach to regular reflections and vice versa. In more detail when the deflection angle is less than $\theta_{v}$ (Von-Neumann criterion) then we have regular reflection. When it reaches the value $\theta_{\mathrm{d}}$ (the detachment angle) we get Mach reflection. Then $\theta$ decreases (deflection angle is the maximum angle), while the shock reflection remains Mach, and when it reaches again $\theta_{v}$ we have conversion to regular [12-14]. This cyclic behaviour denotes that the phenomenon presents hysteresis. Only the transition process is dealt with in this work. 


\section{Solving the flow equations}

\subsection{The mathematical problem}

The flow is described by the Navier-Stokes equations as applied for supersonic flight. The discretized equations must be set up considering the movement of the grid since the flow passes through moving boundaries (walls). For that reason the velocity vector $v$ is equal to the difference of the absolute flow velocity and grid velocity of the moving mesh. The equations are hyperbolic in nature and in an abbreviated form are as follows [4,15-17]:

$$
\begin{aligned}
& \frac{\partial \rho}{\partial t}+\nabla \cdot(\rho \underline{v})=0 \\
& \frac{\partial}{\partial t}(\rho \underline{v})+\nabla(\rho \underline{v v})=-\nabla p+\nabla(\bar{\tau}) \\
& \text { where } \bar{\tau}=\mu\left[\left(\nabla \underline{v}+\nabla \underline{v}^{\mathrm{T}}\right)-\frac{2}{3} \nabla \underline{v} I\right] \\
& \text { tensor) } \\
& \frac{\partial}{\partial t}(\rho E)+\nabla \cdot(\underline{v}(\rho E+p))=\nabla \cdot\left(k_{e f t} \nabla T-(\bar{\tau} \underline{v})\right) \\
& \text { where } E=\mathrm{h}-\frac{p}{\rho}+\frac{v^{2}}{2}
\end{aligned}
$$

The symbols $\mu, \rho, p, \underline{v}, \bar{\tau}, k_{\text {eff }}, \mathrm{h}, T, E$, respectively, denote molecular viscosity, density, static pressure, velocity vector, stress tensor, total thermalconductivity, enthalpy, static temperature and energy.

\subsection{Numerical Solution and Turbulence Modelling}

The above differential equations are solved by the finite volume technique on a Pentium IV machine with a single core and 8 GB maximum available memory. The SIMPLE-Consistent algorithm was chosen for accounting for the pressure-velocity coupling $[18,19]$. Second order upwind scheme was used for the convective terms and central for the diffusion terms. The temporal discretization was of first order.

The formulation for this scheme was fully implicit and the system of the algebraic equations for every time step was solved iteratively by a classic GaussSeidel point iterative method.

For reasons of convergence acceleration, a pseudotime step relaxation was used in every time step with a suitable relaxation factor [19-21]. Furthermore, a classic V-cycle multigrid algorithm was used for the same reason.

Turbulence modelling was used when accounting for the viscous solutions of equations. For that purpose the Spalart-Allmaras model [22,23] was chosen, which solves only one equation, the equation for eddy viscosity. The model was designed initially for low Reynolds numbers but it is used widely for aerospace applications (transonic-supersonicspeeds) [24], despite the fact that its dependent variable is a fictitious one.

The transport equation for the eddy viscosity $v$ is derived by arguments of dimensional analysis and Galilean invariance, and is given by

$$
\begin{aligned}
& \frac{\partial}{\partial t}(\rho v)+\frac{\partial}{\partial x_{i}}\left(\rho v u_{i}\right)=G_{v}+\frac{1}{\sigma_{v}}\left[\frac{\partial}{\partial x_{j}}\left\{(\mu+\rho v) \frac{\partial v}{\partial x_{j}}\right\}+C_{b 2} \rho\left(\frac{\partial v}{\partial x_{j}}\right)^{2}\right]-Y_{v} \\
& \text { (4) } \\
& G_{v=} C_{b 1} \rho S v, S=S_{1}+v f_{u 2} / k^{2} d^{2}, f_{u 2}=1-\frac{\chi}{\not f_{u 1}}, \\
& Y_{v}=C_{w 1} \rho f_{w}\left(\frac{v}{d}\right)^{2}
\end{aligned}
$$

where $G_{v}$ is the production of turbulence viscosity and $Y_{v}$ is the destruction of turbulence viscosity that $v$ occurs in the near-wall region. The symbols $d, S, \chi f_{u 2}, f_{u 1}$ respectively, denote wall distance, deformation tensor, ratio of molecular to kinematic viscosity and functions of molecular and kinematic viscosity. The other symbols are constants.

For the numerical solution of supersonic flow inside the nozzle, two commercial codes were used, FLUENT and GAMBIT (mesh generator), both provided by Fluent Inc. An unstructured mesh of 67000 cells was employed. Inlet conditions were: atmospheric pressure and Mach number of 1.8 and outlet conditions, pressure of 255000 pascal. No slip conditions apply at both nozzle walls and classic wall functions were used $[19,25,26]$.

Density is computed via the perfect gas law (admission of isentropic flow). Finally, concerning the molecular viscosity $\mu$, because during a supersonic flow there exist substantial temperature gradients, a function was defined, which describes the changes of viscosity with respect to temperature. The law of Sutherland [24] was chosen: 


$$
\mu(T)=\mu_{o}(
$$

where $T_{0}=273.16 \mathrm{~K}$ and $\mu_{0}=1.78 \mathrm{kgm}^{-1} \mathrm{~s}^{-1}$ are the reference values.

Due to the movement of the wall boundaries (as they approach each other) the mesh must be updated with time. Specifically the walls move with the rotation speed of 3 degrees per second. The original point of rotation is the edge point of outlet for every wall respectively (Fig.1). As a result it is obvious that the grid is continuously deforming and it has to be updated at every time step. The main techniques applied for updating the deforming mesh is the spring-based smoothing method and local remeshing. The last method has been described in detail in Anthony Anderson's research work [27].

\subsection{Grid refinement and time independency.}

Three different unstructured meshes were generated in order to indicate which mesh produces results independent of further grid refinement. Fig.2 shows the pressure distribution along the axis of symmetry for the three meshes. That of 67000 cells has almost the same distribution of the most refined mesh. The same istrue for the other variables as well. So it was selected for time saving reasons (cpu time).

The second step is the time independency investigation. For this purpose we considered three different time steps $(0.05,0.1$ and $0.2 \mathrm{sec})$. Again pressure distributions are presented at the symmetry plane for three different real time positions. Fig. 3 shows that the choice of time step

$0.1 \mathrm{sec}$ is acceptable.

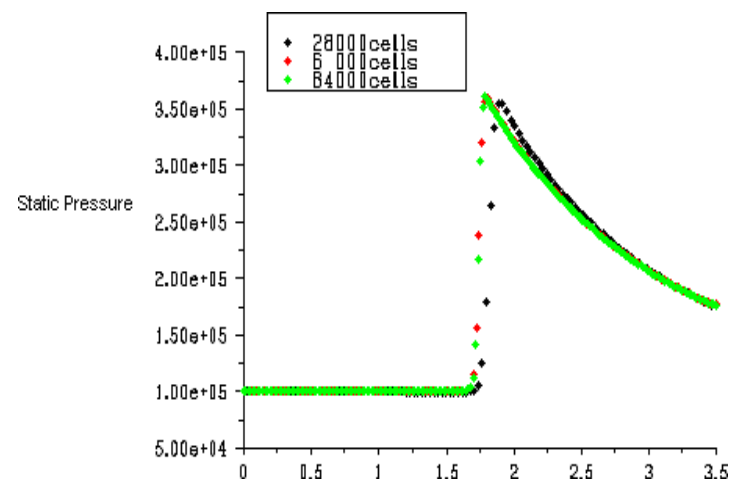

Fig.2. Pressure distribution along axis of symmetry for three different meshes.
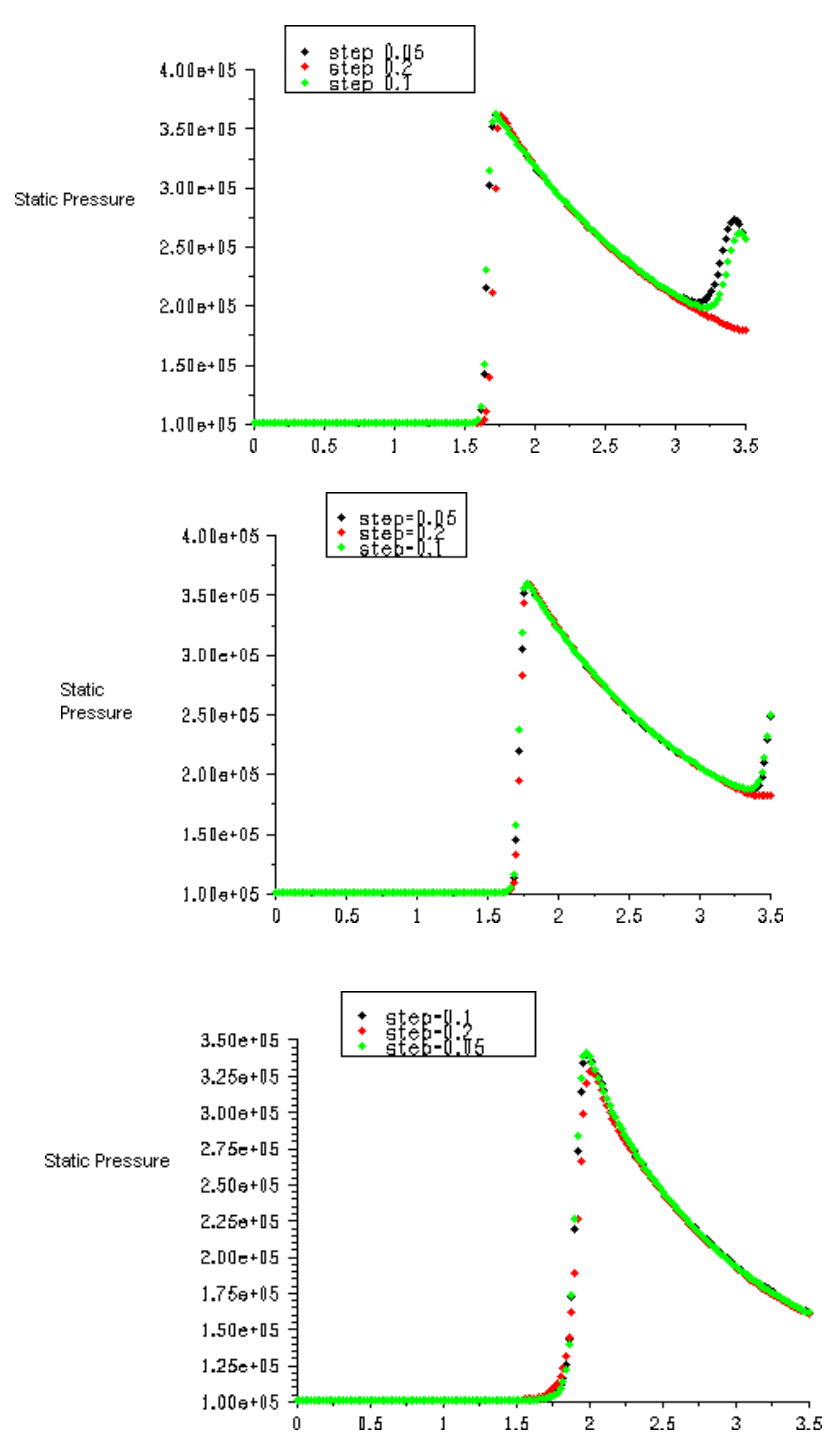

Fig.3. Pressure distribution along axis of symmetry for three different time steps: $\mathrm{t}=0.2 \mathrm{sec}, 0.6 \mathrm{sec}$ and $1.2 \mathrm{sec}$ from top to bottom respectively

\section{Results and discussion}

Computational results were obtained on a Pentium IV Machine with a cpu time of about 6 hours for full convergence of the primitive variables: $\mathrm{x}$ and $\mathrm{y}$ velocity, eddy viscosity and static pressure. Convergence is deemed to occur when the scaled residuals fall below $10 \mathrm{e}-5$. The total number of time steps was 16 , from $0.1 \mathrm{sec}$ to $1.6 \mathrm{sec}$. 
Beginning the analysis of results, and observing at time $\mathrm{t}=0.2 \mathrm{sec}$ the Mach contours, we see the Mach disk formed around the middle of the axis (Fig.4). At the next time steps, we can easily observe both walls approaching each other. As a result, the deflectionangle is decreasing, causing the reduction of disk's length. After 1.5 seconds the Mach disk does no longer exist and the waves intersect at a regular intersection.

Fig. 4 illustrates the presence of an additional process. The disk is moving towards the outlet with time, and the decrease of the deflection angle causes a corresponding decrease to the shock angle of the waves. As a result they become weaker and of course they expand more in the flow domain.
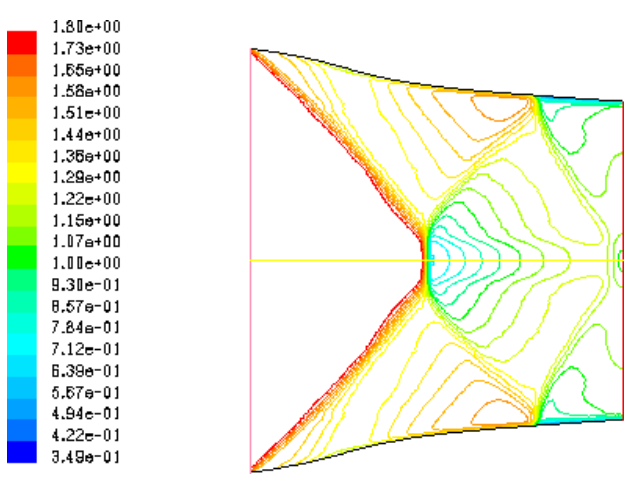

$\mathrm{t}=0.2 \mathrm{sec}$
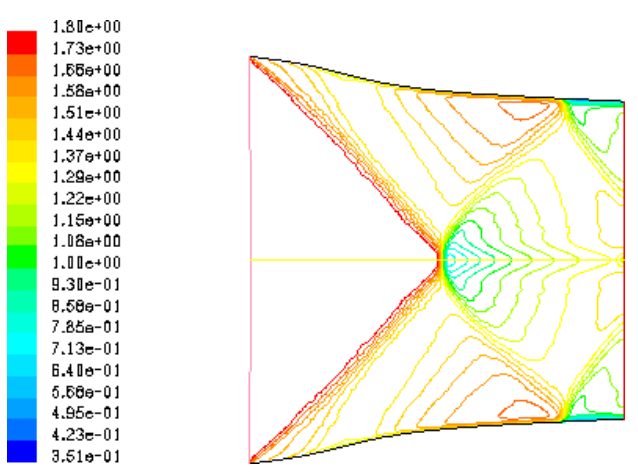

$\mathrm{t}=0.9 \mathrm{sec}$
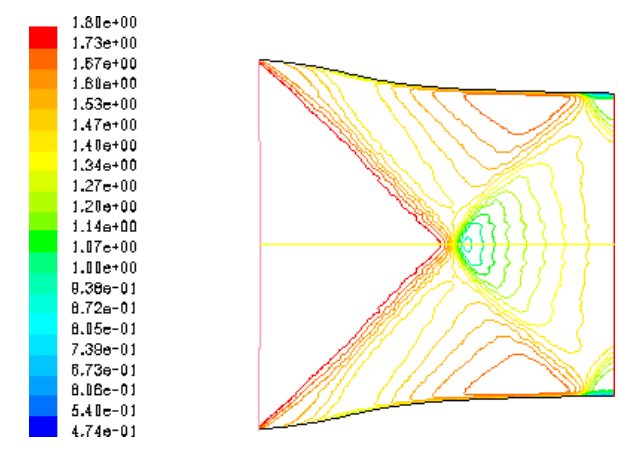

$\mathrm{t}=1.3 \mathrm{sec}$

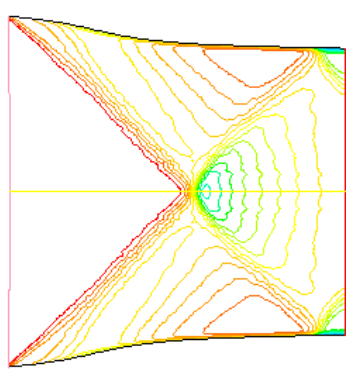

$\mathrm{t}=1.6 \mathrm{sec}$

Fig.4. Mach contours plotted versus time $(\mathrm{t}=0.2 \mathrm{sec}$ to $1.6 \mathrm{sec}$ ).

\subsection{Transition from Mach to Regular intersection.}

In shock wave reflection studies a main objective is to compute the height and position of the Mach disk versus time [27]. In this study, theindependent variable is not the wedge angle, as used in most studies, but time, because of the transient nature of the process. To perform height computation we need information at every time provided by the results obtained until then. Because the Mach disk is a normal shock wave, we trace the region where considerable discontinuities occur (subsonic to supersonic flow). Thiscriterion gives us direct guidelines for evaluating the height value. At first the Mach number distribution at the axis plane is estimated. Fig. 5 displays a significant decrease of Mach number, indicating the position where the Mach disk begins to expand. The first point of this spontaneous change defines the starting point of disk's thickness. The flow there 
remains supersonic. Behind that point, flow becomes subsonic. In this region we take a normal section (direction parallel to the y axis), and we compute the Mach number distribution in this section. We observe that as we move towards the wall (positive y), the Mach number increases (Fig. 6). There is a point where the flow becomes sonic $(M=1)$. After that point the flow returns to be supersonic. The sonic point indicates where the height of the Mach disk ends. This sonic point is the Mach or triple point as mentioned above. Following this process at all time positions, we estimate height as a function of time. Fig. 7shows that height decreases with time and becomes zero (regular reflection) at time $=1.5 \mathrm{sec}$.

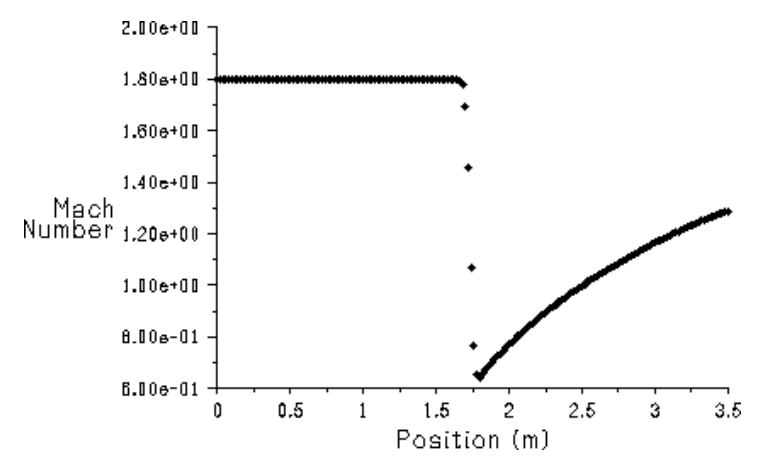

Fig.5 Mach number distribution along the axis of symmetry plane

at a particular time step.

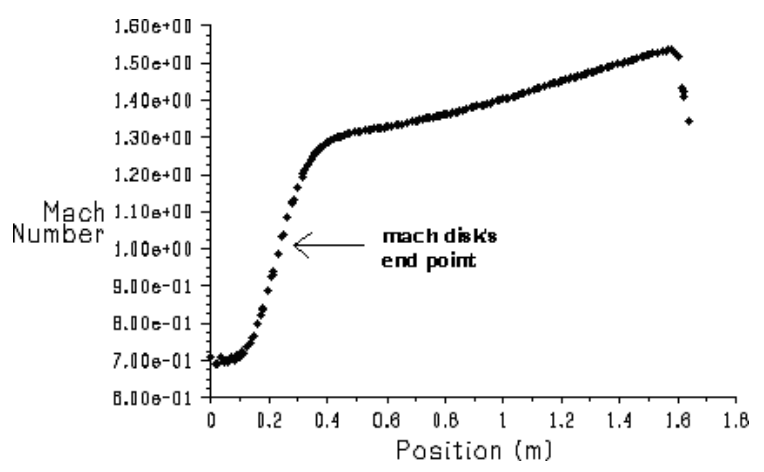

Fig.6. Mach number distribution at the symmetry plane at $\mathrm{x}=1.77 \mathrm{~m}$.

Based on the above procedure, we compute the change of Mach disk's position. From Fig. 6 we find the beginning of disk's thickness. Repeating for the remaining time steps, we plot the disk position versus time (Fig.8). It is obvious that the disk moves towards the outlet.

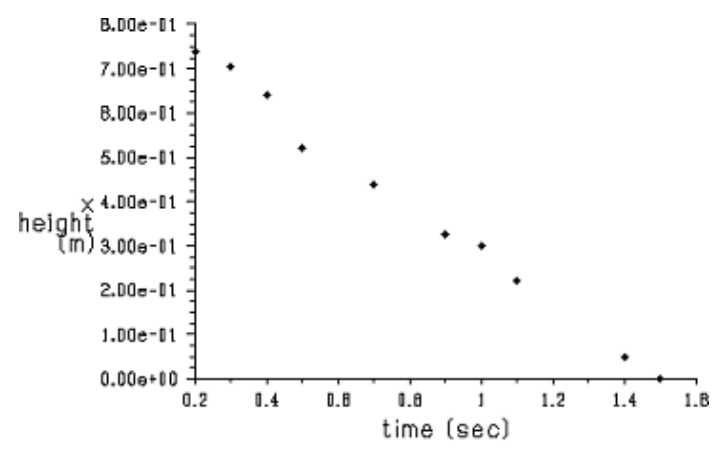

Fig. 7. Disk height varying with time.

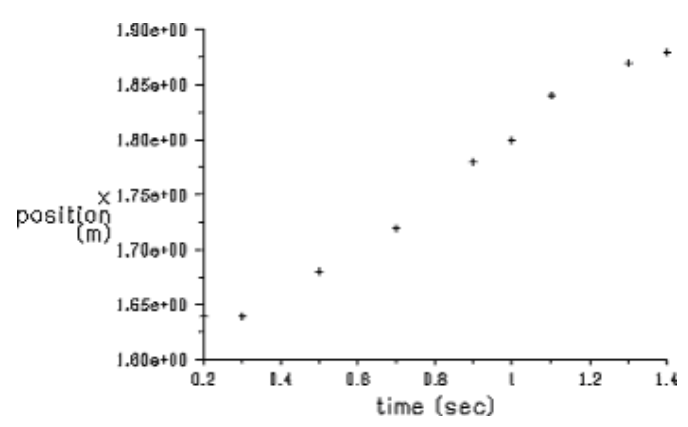

Fig. 8. Disk position along axis of symmetry plotted against time.

\section{Conclusions}

Numerical simulations have been carried out, by means of special numerical and mesh-update techniques. The system equations have been solved by a segregated (not coupled) manner, using known point iterative methods. Flow viscosity was taken into consideration and the full Navier- Stokes equations were solved. The results appear plausible and the shock position and sharpness were predicted in a physically satisfactory manner without the need for special shock capturingtechniques.

Finally, a further interest for numerical research would be to apply periodic movement to the walls, i.e. making them converging and diverging. Then the Mach disk would periodically form and then vanish, depicting the hysteresis process $[7,13,14,28,29]$. 


\section{Acknowledgements}

The contribution of Assistant Professor P.Koytmo (University of Patras), by providingthe license for using the commercial software FLUENT and GAMBIT for the numerical implementation is gratefully acknowledged.

\section{References}

K.Naidoo and B.W.Skews, Dynamic transition from Mach to regular reflection of shock waves in a steady flow, J. Of Fluid Mechanics, vol.750,385-400, July 2014.

[2] M.K.Hryniewicki, J.Gottlieb, C.Groth, Transition boundary between regular and Mach reflections for a moving shock interacting with a wedge in inviscid and polytropic air, Shock Waves 27,523-550, 2017.

[3] J.Gottlieb, M.K. Hryniewicki, C.Groth,Transition boundary between regular and Mach reflections for a moving shock interacting with a wedge in inviscid and polytropic argon, Shock Waves 29, 795-816, 2019.

[4] J.D. Anderson, Supersonic and High Temperature Gas Dynamics, McGraw-Hill, 1989.

[5] Chapman CJ. High Speed Flow, Cambridge Univ. Press (Cambridge Texts in Applied Mathematics, 23), Cambridge, 2000.

[6] Wu, K., Zhang, G. \& Kim, H.D. Study on the Mach and regular reflections of shock wave. J Vis 22, 283-303 (2019). https://doi.org/10.1007/ s12650-018-00542-5

[7] Matheis, J., \& Hickel, S. (2015). On the transition between regular and irregular shock patterns of shockwave/boundary-layer interactions. Journal of Fluid Mechanics, 776, 200-234. doi:10.1017/jfm.2015.319

[8] Chpoun A., Passerel D., Li H., Ben-Dor G., Reconsideration of oblique shock wave reflection in steady flows, J..Fluid. Mech. 1995. Vol.301.P.19-35.

[9] Marie Claude Druguet, David E. Zeitoun, Influence of numerical and viscous dissipation on shock wave reflections in supersonic steady flows, Computers and Fluids, 2003 Vol.32.P.515-533.

[10] Gribben B.J., Badcock K.J., Richards B.E., Numerical study of shock-reflection hysteresis in an underexpanded jet, AIAA J.2000 Vol.38.P.275-283.

[11] Ivanov MS, Markelov GN, Kudryavtsev AN, Gimelshein S., Markelov G., Investigation of the hysteresis phenomena in steady shock reflection using kinetic and continuum methods. Shock Waves 1996;5:341-6.]

[12] Chpoun A., Leclerc E., Experimental investigation of the influence of downstream flow conditions on Mach stem height, Shock Waves 1999;9:269-71.

[13] Dan Wang, Yong Yu, Shock wave configurations and reflection hysteresis outside a planar Laval nozzle, Chinese Journal of Aeronautics, Volume 28, Issue 5, 2015, Pages 1362-1371, ISSN 1000-9361,

https://doi.org/10.1016/j.cja.2015.07.010.

[14] HILLIER, R. (2007). Shock- wave/expansion-wave interactions and the transition between regular and Mach reflection. Journal of Fluid Mechanics, 575, 399-424.

[15] G. K.Batchelor, An Introduction to Fluid Dynamics, Cambridge Univ. Press, Cambridge, England, 1967.

[16] Roman Frolov, An efficient algorithm for the multicomponent compressible Navier- Stokes equations in low- and high-Mach number regimes, Computers \& Fluids, Volume 178, 2019, Pages 15-40, ISSN 0045-7930, https://doi.org/10.1016/ j.compfluid.2018.10.001.

[17] Souverein, L. J., Bakker, P. G. \& Dupont, P. 2013 A scaling analysis for turbulent shock- wave/boundary-layer interactions. J. Fluid Mech. 714, 505-535

[18] Patankar, S. V. and Spalding, D. B., (1972), A Calculation Procedure for Heat, Mass and Momentum Transfer in Three-dimensional Parabolic Flows. International Journal for Heat and Mass Transfer., 15, 1787-1806.

[19] N.C. Markatos, Mathematical modelling of single and two phase flow problems in the process industries, Revue de l'Institut Francais du Petrole, Vol.48,No.6, pp 631-632, 1993.

[20] N.C. Markatos, A.C.H. Mace, D.G. Tatchell Analysis of combustion in recirculating flow for rocket exhausts in supersonic streams, J. Spacecraft and Rocket, Vol.19,No.6, pp 557-563, 1982.

[21] N.C. Markatos, On numerical modelling of embedded subsonic flows, Int. J. Num. Methods in Fluids, Vol.6, pp 103-112, 1986.

[22] P.R. Spalart and S.R. Allmaras, A one- equation turbulence model for aerodynamic flows, Technical Report AIAA-92-0439, American Institute of Aeronautics and Astronautics, 1992.

[23] P.R. Spalart, S.R. Allmaras, A one equation turbulence model for aerodynamic flows, AIAA, 1999.

[24] Sebastian Deck, Philippe Duvaeu, Paulo d'Espincy, Philippe Guillen, Development and application of SpalartAllmaras one equation turbulence model to three dimensional supersonic complex configurations, Aerospace Science and Technology pp. 171-183, January 2002.

[25] B.E Launcher and Spalding D.B., The numerical computation of turbulent flows, Computer Methods in Applied Mechanics and Engineering, 3:269-289, 1974.

[26] John C.Tannehill, Dale A.Anderson, Richard H.Pletcher, Computational Fluid Mechanics and Heat Transfer, Taylor \& Francis Inc, 1997.

[27] Anthony Anderson, Xiaoming Zheng and Vittorio Cristini, Adaptive unstructured volume remeshing, Journal of Computational Physics, Volume 208, Issue 2, 20 September 2005

[28] E.Koronio, G.Ben-Dor, Oren Sadot, M.Geva, Similarity in Mach stem evolution and termination in unsteady shock- wave reflection, J. of Fluid Mechanics, Nov.2020, vol.902,A1.doi:10.1017/jfm.2020.540.

[29] Kexin Wu, Guang Zhang, Heuy Dong Kim, Study on the Mach and regular reflections of shock wave, J. of Visualization, 22,283-303, 2019.

\section{Creative Commons Attribution License 4.0 (Attribution 4.0 International, CC BY 4.0)}

This article is published under the terms of the Creative Commons Attribution License 4.0 https://creativecommons.org/licenses/by/4.0/deed.en_ $\underline{\mathrm{US}}$ 